\title{
Equipment issues regarding the collection of video data for research
}

\author{
Rebecca Lippmann Kung, ${ }^{1, *}$ Peter Kung, ${ }^{1}$ and Cedric Linder ${ }^{1,2}$ \\ ${ }^{1}$ Fysiska Institutionen, Uppsala Universitet, Uppsala, S 75121, Sweden \\ ${ }^{2}$ Department of Physics, University of the Western Cape, 7535 Cape Town, South Aftica
}

(Received 11 May 2005; published 31 October 2005)

\begin{abstract}
Physics education research increasingly makes use of video data for analysis of student learning and teaching practice. Collection of these data is conceptually simple but execution is often fraught with costly and time-consuming complications. This pragmatic paper discusses the development of systems to record and permanently archive audio and video data in real-time. We focus on a system based upon consumer video DVD recorders, but also give an overview of other technologies and detail issues common to all systems. We detail common yet unexpected complications, particularly with regard to sound quality and compatibility with transcription software. Information specific to fixed and transportable systems, other technology options, and generic and specific equipment recommendations are given in supplemental appendices
\end{abstract}

DOI: 10.1103/PhysRevSTPER.1.010105

PACS number(s): 07.07.Hj, 07.05.Hd, 01.40.Fk

\section{INTRODUCTION}

Video data have revolutionized research in education, making new forms of analysis possible. Transcription and analysis from video digitally contained on a computer allow consistent time stamping, immediate searching and scanning, and portability of data. This method has also, however, placed a sizable burden of video collection, preparation, and archiving upon the shoulders of education research groups. Considering the time and intellectual investment in video analysis, it is critical that a group's video recording and archiving system be well planned, usable, and reliable. Any improvement in process efficiency reaps multiple benefits for the quality and expediency of research.

This paper proposes the use of consumer DVD video recorders to capture and permanently archive multiple video and stereo audio tracks in a form usable by PC-based transcription programs. Many other solutions require the video data to be processed (imported and/or compressed) prior to transcribing and archiving, doubling or tripling the time involved. With this system "real-time" recording is possible, requiring negligible preparation time for use with commonly available transcription software.

This paper begins by highlighting likely requirements for a video recording system in a teaching and learning environment, and indicates why we feel a system based around consumer DVD video recorders best fulfills those needs, whether the installation is temporary or semipermanent. Next, the two primary decision points of digital vs analog video and microphone type are covered, and then more detailed component and setup recommendations are made. This section also details our potentially nonobvious method of capturing MPEG2 data from DVD video discs and the resulting issues.

The brief overviews of other feasible video-to-PC technologies and the discussion of integration with the transcription software TRANSANA contained in the Appendixes may be useful to research groups with needs different from our own. Further suggestions applicable to transportable and multiple camera installed systems are also contained in the appendix.

We do not intend this to be a step-by-step recipe for a ready-made recording system, but we do offer a very pragmatic presentation of the options and pitfalls that may be encountered even if a different technology is used. It is imperative that you conduct your own research on technology options, availability, and costs. With the rate of change in this field, it is essential to use this paper as merely an introduction to the issues and options rather than a shopping list.

\section{POTENTIAL REQUIREMENTS AND NEEDS}

While no two education research groups will have identical requirements, there are several aspects that all must consider. We believe that the following assumptions and requirements are most critical for a successful recording system for transcription in an educational environment.

\section{A. Compatibility with analysis software}

The archived audio and video must operate logically and consistently with the chosen analysis software, ideally without copying the data to the host computer's local hard drive.

\section{B. Compatibility with accepted standards}

The archive format must be readable with a minimum of proprietary hardware or software and be able to be copied and distributed freely. While the primary goal of the system is only for internal transcription and/or analysis, we do not wish to preclude sharing and presenting video data with and to others. Likewise, it is important that the recording remain readable for several years from both physical and technological standpoints.

\section{Ease of use}

Ease of use is absolutely essential. An operator in addition to the class teacher or lecturer is a luxury that is rarely 
available. Because of this, the system must be capable of at least $2 \mathrm{~h}$ of continuous unattended recording. Changing recording media is at best disruptive and at worst impossible. Likewise, ac power is preferred to batteries and other consumables. The operator should have to do little more than press a few buttons to begin recording.

A minimum of postprocessing should be required to convert the data into a usable form. At the conclusion of the class, data should be nearly ready to transcribe. Any postprocessing, no matter how simple, can become a surprisingly heavy burden to the researcher.

Although proper documentation is essential, we would like the system to be conceptually understandable on its own. Highly proprietary interfaces and devices should be avoided when possible, and there should be logic and consistency to interfaces and conventions.

\section{Audio and video quality}

High audio quality is the foremost enabler of successful transcription or analysis. This includes not only capability of the recording media, but also the clarity of speech and absence of buzzes, cracks, hisses, and other distractions. Previous experience has shown that a stereo audio track drastically eases transcription of a group setting and is essential if there are multiple speakers in close proximity.

Video quality must be sufficient to determine who is speaking, see facial gestures, and follow the general work process in, for example, a laboratory class, and perhaps see large-group presentation materials. Capturing the text of students' individual papers is not a primary goal.

\section{E. Flexibility}

The basic requirements of the system will ideally not be bound by a specific room installation. While many research groups will install hardware into one specific location, many others will need compatible systems in a transportable form (see Appendix D). The layout should also be flexible enough to accommodate varying quantities and qualities of video and audio. One may need several cameras recording different groups of students at the same time, providing multiple simultaneous video and audio tracks. At other times multiple views of a single group of students will be most useful.

The system should be open for future upgrades, expansion, or alterations as well as any required repairs or component replacement.

\section{F. Recording system presence}

The visibility of cameras and microphones is partly an ethical issue, and anyone performing research on human subjects should have the oversight of a human subjects review board. (At the very least, written consent from the subjects will be required.) We do not recommend completely concealing the recording system, but interruptions in the normal flow of work will negatively affect data. Students should not have to speak towards specific areas or face certain directions. Individual lavalier microphones cannot be placed on the students, unless in a formal presentation setting. Microphones, cables, and other hardware should be as unobtrusive as possible and require a minimum of setup and everyday maintenance.

\section{G. Costs}

Current realities dictate careful consideration of the costs not only of initial setup, but also of media, maintenance, training, and upgrades. The ability to use the system for other research or education purposes, such as video playback and analysis, may also be appreciated.

\section{BENEFITS OF DVD VIDEO RECORDERS}

After considering the above requirements and thoroughly researching numerous technology options, some described in Appendix B, we determined that a system utilizing consumer DVD video recorders currently provides the best technological basis for simple education research recording.

Conceptually evolved from the VCR, DVD video recorders write video and stereo audio to a recordable or rerecordable DVD. Using a form of the MPEG2 compression format, they record in real time between 1 and $8 \mathrm{~h}$ of video on a single-layer 4.7 gigabyte disc. They are designed for use with one's television and home audio system and are typically sold through home-electronics retailers. We selected this technology for the following reasons.

\section{A. Compatibility with transcription software}

The specific MPEG2 streams produced by some of these recorders are readable by our software and the general file format is standardized and very widely accepted. Recordable and re-recordable DVDs are affordable and available. With minor accommodations the MPEG data from some recorders can be read into most computer programs including our chosen transcription software.

Note that this video format is not that used by most video editing software. Thus, a decoding step (software available for little or no cost) is required before adding titles, transitions, obscuring subjects' identities, or other editing functions.

\section{B. Compatibility with accepted standards}

Used by literally millions of consumer DVD video players and computers, this file format is established, standardized, and will be supported for decades to come. Every major computer operating system supports the format in some manner. Even if the specific method of reading data directly from the disc into the transcription software becomes obsolete, the data will always be available to a stand-alone DVD player for transfer to a superceding format.

Under ideal conditions, a write-once DVD disc can last up to $100 \mathrm{yr},{ }^{1}$ and newer scratch-resistant and uv-protected DVDs better resist incidental damage. Even in far from ideal conditions, we expect the optical media to have a greater lifespan than most forms of magnetic media. 


\section{Ease of use}

DVD video recorders are designed for consumer home use and thus use familiar controls and terminology. There are no computer booting or software configuration delays and if desired the recorders can be set to start and stop recording automatically, allowing completely unattended operation. There are no batteries to be replaced and a single disc can record for up to $8 \mathrm{~h}$, typically eliminating the need for changing media. Prior to use in a computer, the only step (other than the physical removal of the disc from the DVD recorder and labeling) is the finalization of the disc. This process, which can be done at any time after recording, requires only a few button presses and typically between 1 and 3 min; DVD+RW (plus rewritable) discs do not even require this step.

\section{Audio and video quality}

Stereo audio quality is comparable to that of a compact disc. Although the longer recording times do compress the audio tracks, environmental noises such as fans, coughs, and paper rustling far exceed any compression artifacts under all but the most ideal conditions.

Video is captured at a resolution of up to $720 \times 480$ (NTSC) or $720 \times 576$ (PAL), although increasing recording time per disc will correspondingly reduce picture quality. We find that DVD video's highest resolution is often unnecessary when transcribing and reduce the size of the video playback window. We zoom in only if there is a specific item of interest.

\section{E. Flexibility}

A DVD video recorder can use a variety of standard ana$\log$ and digital video inputs. If needed, this allows easy recording of many other sources, such as other cameras, computers, document cameras, or whiteboard scanners. Video and audio switchers, splitters, and mixers are commonly available which allow different sources to be recorded consecutively or simultaneously to the same or multiple discs as the situation requires.

In multiple-camera setups, each camera can receive its own DVD video recorder and record onto its own disc, independent of any central coordinating device. Coordinated functions, such as power on, starting, and stopping, can be accomplished by using identical DVD recorders operating on the same remote-control ir frequency. With the exception of loading and labeling discs, six recorders require no more work by the operator than one.

\section{F. Recording system presence}

A DVD video recorder is not permanently connected to the camera and microphones. It can be placed physically distant from the cameras and microphones, potentially in a separate room. Operation via remote control can allow even less obtrusive use if desired.

\section{G. Costs}

Already quite affordable in comparison to computer-based solutions, both the DVD recorders and the media are likely to become even more affordable with time. Standardized interfaces allow simple upgrades and quick repairs without requiring specialized training. Maintenance can be limited to items such as replacing remote control batteries once or twice a year.

\section{SYSTEM SETUP CONSIDERATIONS}

Systems based around DVD video recorders or similar digital recording devices are most affected by two primary decisions. First is the transfer of video between the camera and the DVD recorder via digital cables or analog cables. The second major decision regards the use of microphones which can plug directly into the camcorder's microphone port or microphones which need additional hardware for use.

\section{A. Digital vs analog video}

Video data can be transmitted between the camcorder and the DVD recorder either digitally, using a Firewire cable (also known as iLink or IEEE1394), or via an analog S-video (SVHS) or RCA cable. Because specialized cameras, DVD recorders, and other hardware are needed for digital signals, this decision should be made early in the planning process although a digital system can be relatively simply converted to analog and the two can coexist.

Digital video can provide a sharper image and brighter colors, but also requires higher-end DVD recorders, DV cameras, Firewire hubs, and potentially microphone conversion devices. There is a noticeable improvement in image quality and color saturation as shown in Figs. 1-4, but no quantifiable improvement in audio quality and even degradation in some cases.

Although a single Firewire cable carries both audio and video, the wiring becomes complicated for any cable runs longer than $5 \mathrm{~m}$ since a powered hub or active repeater cable is required. The active Firewire repeater cables available, although not requiring power from a wall outlet, do need an active power source somewhere in the Firewire chain. Since most cameras and DVD recorders use four-pin unpowered ports, an ac-powered hub and associated six- to four-pin cables or adapters will be required for any run over $5 \mathrm{~m}$. Video quality remains consistent regardless of digital cable length.

Analog S-video cable needs no repeaters and is readily available in lengths up to and beyond $25 \mathrm{~m}$. Longer cables are more susceptible to interference and image degradation, however. Separate stereo audio cables are required, for a total of three interconnects between the camcorder and the DVD video recorder.

The clarity of a digital system is most useful if one needs to read students' documents, but consider simply photocopying or scanning the papers if this is required. For general transcription work, analog systems are usually adequate.

\section{B. Consumer vs professional microphones}

In this discussion we will group microphones into two basic categories: consumer and professional microphones. 


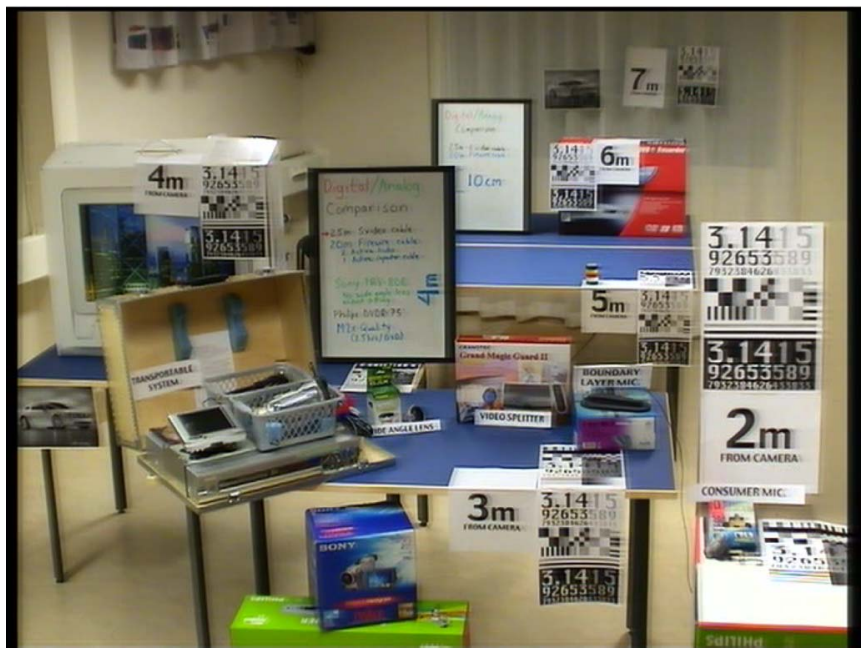

FIG. 1. (Color) Analog video quality. $2.5 \mathrm{~h}$ per DVD, $25 \mathrm{~m}$ S-video cable.

Consumer microphones connect via an unbalanced tip-ring sleeve (TRS or "miniheadphone") connector, and professsional microphones use balanced cables and, generally, XLR-type connectors.

\section{Consumer microphones}

Consumer microphones can plug directly into a camcorder's microphone input and in many cases draw power from it. Consumer microphones' biggest drawback are their unbalanced cables. These have no interference canceling and a lower nominal power level, making longer cables susceptible to humming and buzzing noises, at times serious enough to make transcription impossible. The severity of this interference is dependent upon the length and physical location of the cable (proximity to fluorescent light bulbs, etc.).

Specifications for consumer microphones are often not published or are incomplete, and the marketing terms are often not consistent with industry or scientific standards. Socalled zoom microphones, for example, typically do not

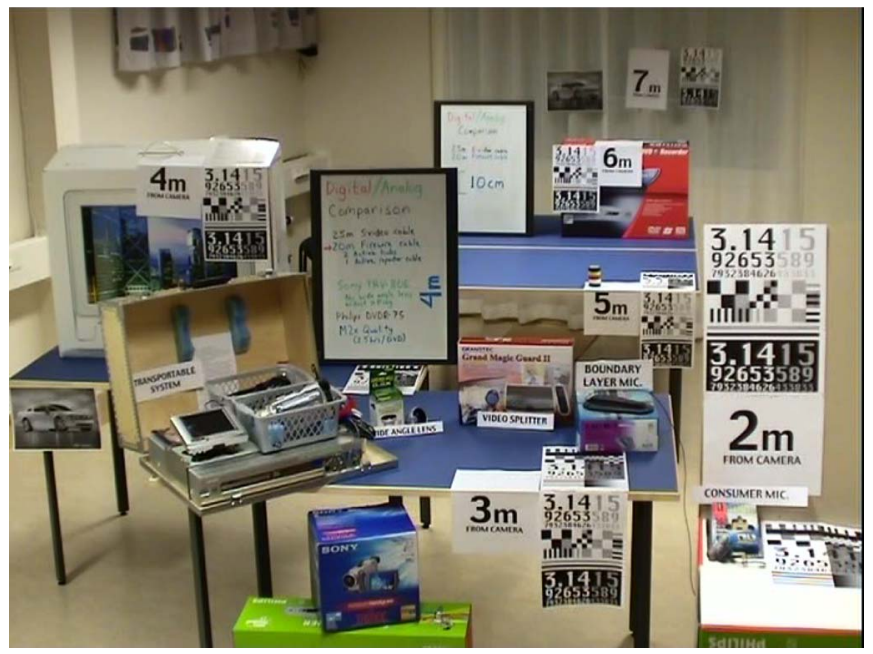

FIG. 2. (Color) Digital video quality. $2.5 \mathrm{~h}$ per DVD.

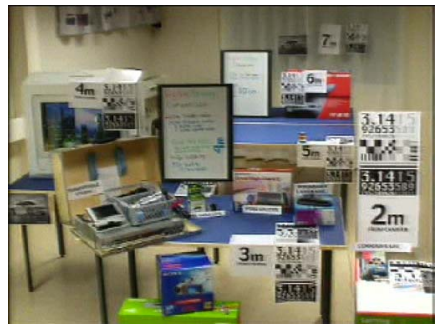

FIG. 3. (Color) Analog video quality. 6 h per DVD.

zoom the sound pickup in any way, but rather mix the external microphone's input with the camera's built-in microphone; these should be avoided unless this mixing effect can be disabled.

\section{Professional microphones}

The balanced cables of professional microphones, used for everything from church amplification systems to Hollywood movie productions and rock concerts, rarely show interference that will be perceptible to this paper's audience. Thus, microphones can be optimally placed anywhere in a room. These microphones, however, cannot plug directly into low- and mid-range consumer camcorders. An XLR to TRS adapter box or another stand-alone device such as a mixer must be used between the microphone and the camera (with a very short unbalanced cable into the camera). Most of these microphones are powered by "phantom power" which also must be provided by the adapter box.

There is a wide variety of professional microphones available, and specifications are easily obtainable. Low-end professional microphones are comparable in cost to high-end consumer versions, although most do not capture singlepoint stereo, doubling the number of microphones required in many transcription setups. There is no limit to the cost, and corresponding quality, of professional microphones, but this audience will probably find quite acceptable results in the low end of the professional microphone spectrum. Professional microphones can also be rented, for occasional use or experimentation purposes.

Complicated setups, such as those involving multiple small groups in close proximity, may require the wider range and flexibility of professional directional microphones.

Many headaches can also be avoided by using professional microphones in any situation which requires the camera to be physically distant from the microphone.

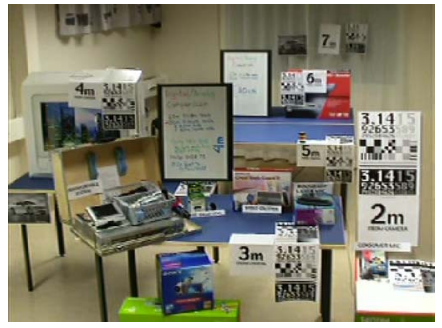

FIG. 4. (Color) Digital video quality. $6 \mathrm{~h}$ per DVD. 


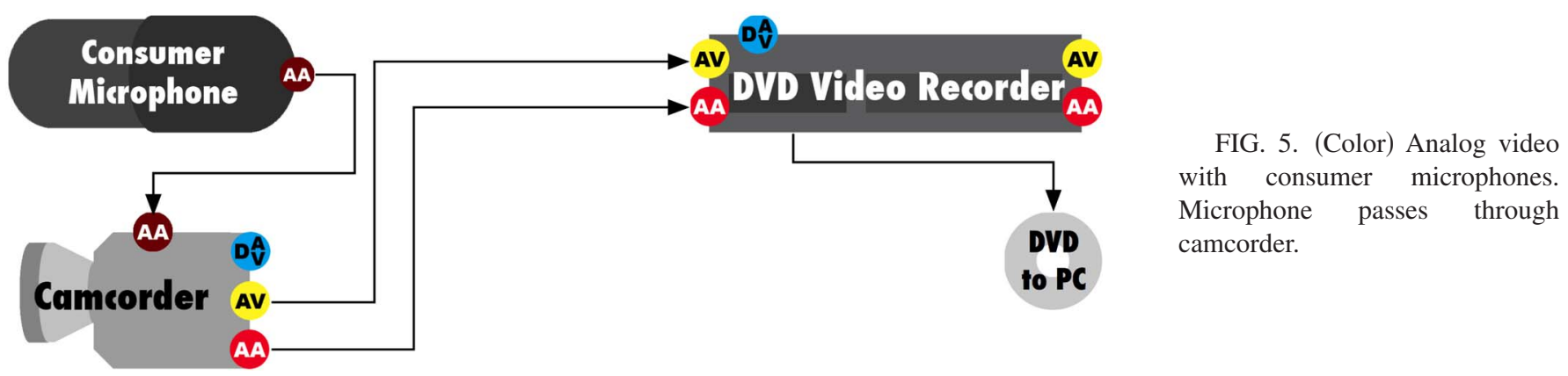

\section{COMPONENT LAYOUT}

\section{A. Component layout with consumer microphones}

With consumer microphones and analog video, audio typically will travel through the camera to the DVD recorder, as shown in Fig. 5, although other amplification devices could be used in place of the camcorder if desired. With digital video, the audio must pass through the camera as in Fig. 6, as DV audio and video signals are not easily separable.

We recommend running the microphones through the camera, if the physical layout allows short unbalanced cables. There is no firm rule for acceptable unbalanced microphone cable lengths, but we have personally had some success with lengths up to $8 \mathrm{~m}$ using careful shielding and cable construction. Running the ground through its own conductor and connecting to the cable's shield at only one end seems to produce the best results. ${ }^{2}$

If the audio cannot be run through the camera, other devices (such as a karaoke mixer, consumer tape decks and receivers, do-it-yourself amplifiers, or even another camcorder) can convert a consumer microphone's mic level input to a line level output for connection directly to the DVD recorder, bypassing the camera. A line level signal is less affected by interference. In most home setups line level cables are not even shielded, although we would recommend against this in most classroom-style environments. Premade, long, well-shielded line level cables are often available from aftermarket car audio system retailers, for use with trunkmounted amplifiers. Most camcorders provide the microphone with plug-in power which eliminates the need for batteries or yet another device to power a consumer microphone.

\section{B. Component layout with professional microphones}

Professional microphones, although not suffering from cable interference issues, must accommodate a converter between the microphones' balanced XLR outputs and either the cameras or the DVD recorder's unbalanced inputs. Again, a camcorder will need a mic level input, and the DVD recorder will expect a line level input.

Like the consumer microphone setup, with analog video, the XLR converter box or mixer can sit between the microphones and DVD recorder as shown in Fig. 7, bypassing the camera. In our setup, audio and video remain synchronized when the camera's sound is not used, and this is probably the simplest, most reliable way to achieve good sound with professional microphones.

It is more complicated and more expensive to use digital video and professional microphones since audio must be routed through the camera, as shown in Fig. 8. In our own experiments, we found unacceptable hum from a cable of about $10 \mathrm{~m}$ between the mixer output and a camera input. This interference (coupled with what seemed to be a lag in our camcorder's automatic gain control as discussed in the camera audio section) rendered the audio unusable. This result may be specific only to our room and equipment.

When using a mixer or other device not specifically designed to work with a camcorder, a resistive attenuator is needed to reduce the mixer's line level output to microphone level. We had hoped to reduce the interference in our experimental system by placing the attenuator near the camera's input, but the noise remained unacceptable.

Individual purpose-built camcorder XLR adapter boxes would solve the noise problem, eliminate the need for the attenuator, and provide less opportunity for improper settings. These XLR converter boxes are commonly used in videographers' field setups and work particularly well if a
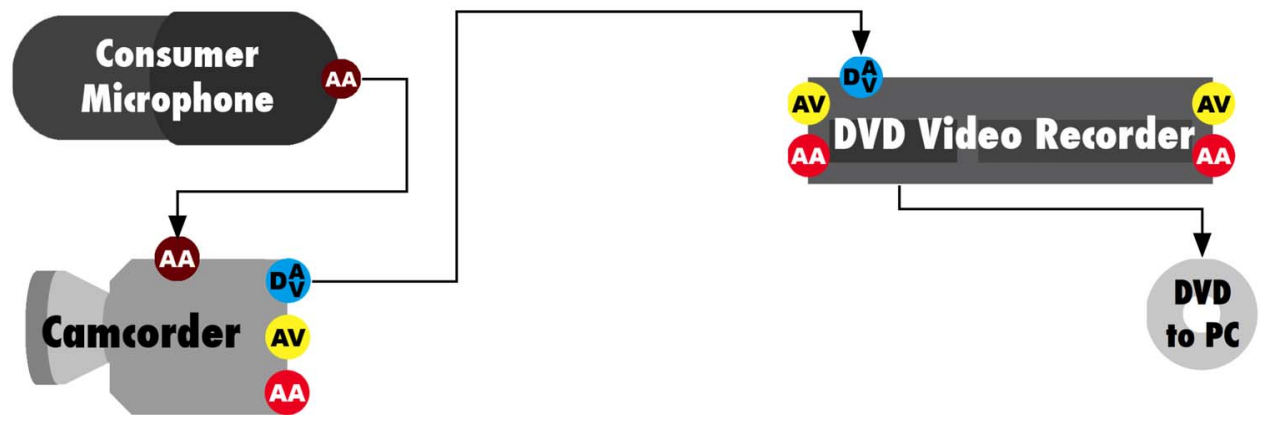

FIG. 6. (Color) Digital video with consumer microphones. Audio passes through camera, and both video and audio pass via a Firewire cable to the DVD recorder. 


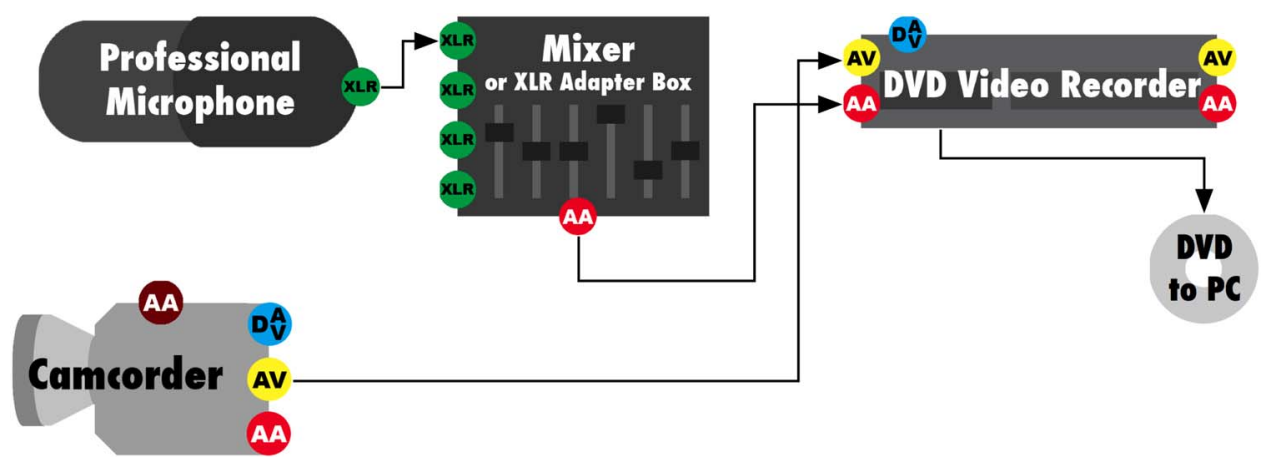

FIG. 7. (Color) Analog video with professional microphones. Audio does not pass through camera. Analog video and audio pass through separate cables to the DVD recorder. camera's automatic gain control feature can be disabled. These higher-end cameras and adapter boxes which can selfpower professional microphones unfortunately both add significant cost.

\section{HARDWARE CONSIDERATIONS}

\section{A. Cameras}

Surprisingly, the camera is one of the least critical components in the chain. Most consumer camcorders have acceptable image quality in well-lit, stable conditions such as most classrooms. Many setups will not utilize any of the tape or recording functions, and indeed could be well served by a camera without those functions. In many instances security cameras and other camera-only devices can be used, but do not assume that their focus, zoom, picture quality, and ease of use will be as consistently acceptable as those of consumer camcorders.

Manual exposure and white balance controls prove useful in situations where the background lighting changes behind the transcription subject, such as windows or an overhead projector. Changing these settings in the middle of a recording session can be disruptive or logistically difficult, so the best solution is often to simply choose lighting and angles which are friendly to autoexposure systems. Optimization of lighting and manual color settings is well described in resources for home and amateur videographers.

It is essential that the camera operate for long periods of time in standby mode, without recording but only providing a video output signal to the DVD recorder. Some cameras may begin a demonstration mode or turn off completely after a certain amount of time. Ensure that this can be disabled.
If planning to use a videotape as a backup, verify that the tape can be changed while mounted to a tripod. This is increasingly an issue as camcorders become smaller. Likewise, a convenient power switch or remote will also prove beneficial.

\section{Cameras with audio}

When using digital video, audio must pass through the camera (Figs. 6 and 8). Under most circumstances, consumer microphones will also connect directly to the camera (Figs. 5 and 6). For these cases, the most important qualities of the camera relate to audio performance. Most consumer camcorders feature automatic gain control (AGC), sometimes called automatic level control. This varies the gain on the sound input, turning down loud noises and amplifying soft ones. While useful in some instances, when used with an external microphone we found the AGC to produce an irritating hiss during quiet periods and what seemed to be an occasional sound adjustment lag. The lag was more pronounced with professional microphones than their consumer counterparts, but is likely to vary with different camera models. A camera with a manual input volume control will produce more consistent audio from which to transcribe, since the sound levels in most classrooms are generally known and automatic adjustment is unnecessary.

Cameras that provide balanced professional microphone inputs (XLR connectors) and phantom power would allow the professional microphones to be used directly, but their cost is significantly higher due to other advanced features which will go unused in most setups.

Nearly all camcorders have plug-in power, which provides a low voltage on the $3.5 \mathrm{~mm}$ microphone jack to power
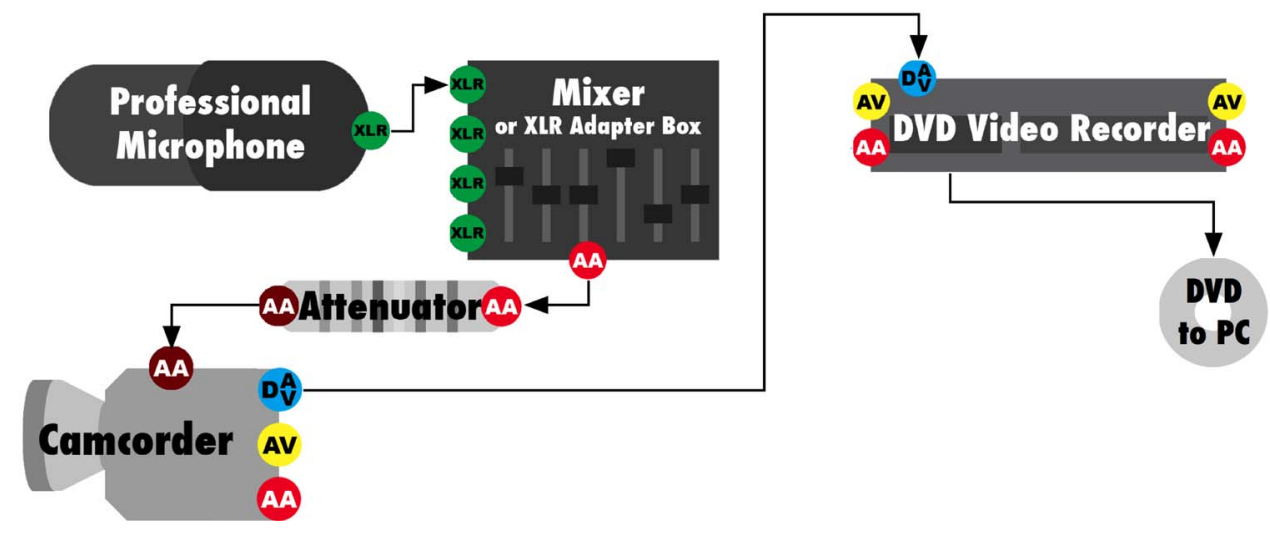

FIG. 8. (Color) Digital video with professional microphones. Audio passes through camera, and both digital video and audio pass via a Firewire cable to the DVD recorder. 
consumer microphones without batteries. Microphones using this standard $3.5 \mathrm{~mm}$ stereo microphone jack are usually not brand specific. A Sony microphone, for example, should work with a Canon camera. This is not the case for powered accessory shoe microphones which work only with some brands or some models of cameras.

\section{Cameras without audio}

When using analog video signals and professional microphones, there is no reason to pass audio through the camera (Fig. 7). Any autofocus video camera or camcorder with the proper video-out signal should suffice. In theory an S-video output provides slightly better quality than an RCA-style jack.

\section{B. Microphones and microphone placement}

There are numerous usable microphone setups, varying in style, pickup pattern, quantity, and placement, all of which must be individually optimized for each venue and occasion. No amount of postprocessing or enhancement can replace good-quality initial sound capture.

In an environment with only one person speaking at a time, even the on-camera microphone is passable although not ideal. When the speaker can be anywhere in the room, numerous omnidirectional microphones, often designed for conference or podium recordings, exist and can provide outstanding results in these simple setups.

Successfully recording students working in multiple small groups in a single room is a much harder situation. It is more critical to get the microphone close to the group, and the microphone may require some directionality to exclude other groups. Unfortunately, no microphone is truly directional, able to pick up sound from only one direction. Rather, directional microphones change the tonal quality of off-axis noises. In our case, this can be used as an advantage when transcribing. The transcriber can learn to discern and ignore sounds coming from off-axis directions.

There are two general strategies for microphone placement in a small-group setting. One clusters the students around a low-directionality microphone in the center of a table or similar, and the other attempts to be less obtrusive by using a more directional microphone further away from the students.

Placing a microphone in the center of the table is theoretically ideal, but every pen tap, table bump, and paper shuffle is also amplified. Padding and mounts can mitigate this somewhat, but a microphone remains a tempting target for student fiddling.

Highly directional microphones mounted up to $2-3 \mathrm{~m}$ away from the students could allow sound pickup to be targeted at a specific group of students. The use of directional microphones (hypercardiods and short shotguns, etc.) indoors is often discouraged due to unflattering voice pickup and reverberations. Little investigation has been done for a situation such as transcription where speech intelligibility rather than sound accuracy is paramount.

A potentially promising solution in many situations is to combine the proximity of the tabletop solution with the di- rectionality of the shotgun microphone by hanging a microphone from the ceiling, just out of arm's reach. This would be similar to a boom microphone used on a movie or television set although still farther away from the speaker. This could be a promising arrangement if the sound absorption characteristics of the room-particularly the floor and tables-are sufficient. Hanging microphones are often used in courtroom settings, and this may be a good related area to examine.

Note that ringing mobile phones can cause a series of beeps in the microphone system, even if the telephones' ringers are not audible. Thankfully, speech remains mostly intelligible although the beeping quickly proves irritating.

\section{DVD video recorders}

\section{Software compatibility}

Most critical in the selection of a DVD video recorder is its file compatibility with the chosen analysis software. Not all recorders produce data usable in all software programs, although perfectly readable by hardware and some other software programs. This information cannot be found on a specification sheet; a test recording must be made.

Many software programs will not read DVDs directly but will read MPEG2 video. DVD video is a specialized form of MPEG2, and with the proper parameters raw data files can be read into the transcription software, unmodified save for perhaps the file extension.

A DVD video recorder places video and sound data into a series of video object set (VOB) files (titled Video_01.VOB or similar). Information such as menu structure, chapter breaks, titles, and language and angle options is saved in another series of files with the IOB extension. DVD video players use the file length information contained in these IOB files. In order for us to take the DVD directly from the recorder into an analysis program, we treat the VOB files as MPEG files. (See Appendix A.) When we disguise the VOB file as a typical MPEG file, the computer must use the file length information in the VOB file.

We have found some DVD video recorders that seem to provide improper time information in the VOB file. They work perfectly when used as a video DVD, but when the VOB file alone is read into an MPEG player, the file ends early. Since one is not technically supposed to be hijacking the data this way, there is no reason for the DVD recorder producer to ensure that the VOB files have "proper" time data. Before investing in a specific DVD video recorder to compress MPEG, it is essential that a test disc be recorded and played in your transcription software.

A second issue with using video data directly from VOB files concerns the file breaks. The maximum file size permitted on a DVD is one gigabyte. Since a single-sided DVD holds 4.7 gigabytes, multiple files are needed. Some recorders fill a VOB file until it reaches 1 gigabyte, while others take the total quantity of data recorded onto a DVD and distribute it among five files, even if much smaller than 1 gigabyte. A DVD with only 500 megabytes on it would have five 100 megabyte VOB files. As with the time issue, this is of no consequence to a video player as all the needed 
title and chapter information is in the IOB file and data are switched between VOB files invisibly. We, however, need to open a new data file in our analysis software each time a VOB files breaks, which could prove irritating if the break is mid-sentence or mid-word. Therefore, we prefer files to fill to 1 gigabyte before beginning another file. With a recorder that breaks the disc into five files, a transcriber working with a DVD with only $5 \mathrm{~min}$ of video will have to change files every minute. Again, the only solution is to burn a test disc before investing in a particular make and model.

Some recorders also begin a new set of VOB files each time the user starts and stops recording. This is probably most convenient to a transcriber. Other recorders write a new recording to one of the disc's existing five VOB files. Thus, the start and stop of VOB files do not correspond to a specific recording session. Any recording speed differences in a subsequent recording session may render the affected VOB file unreadable to the transcription software (although no data are irretrievably lost; they can always be read by a DVD player or DVD software and reencoded). Later recording sessions may also be placed before earlier ones. For these recorders, we recommend pressing start and stop only once per disc and filling the disc as much as possible.

\section{Other DVD recorder considerations}

Beneficial features which can be easily inspected are extensive front panel controls, a menu system usable without a TV hookup, and an eject button on the remote. All of these ease work flow and disc switching. The ability to set multiple remote-control frequencies could be useful for operating only part of a multiple-recorder system, although covering selected infrared receivers is effective as well. Input ports, including an S-video input if using analog video, on the rear of the recorders allow much cleaner wiring and a lower chance of a cable accidentally being dislodged. Were they to exist, rear Firewire ports would also be useful but we have not seen any recorders thus equipped. For a transportable system, the retention of clock and setting information when unplugged would also be appreciated.

Some recorders feature a setting to record $8 \mathrm{~h}$ per disc. This is accomplished through audio compression, not through further compression of the video, so this setting will not always be ideal for transcription use.

\section{Wide angle lenses}

Due to the constrained physical layout in many classrooms and laboratory rooms (and potentially a desire to keep the camera as close as possible to consumer microphones) wide-angle lenses may be needed. Technically, these are wide-angle "converters" or "conversion lenses" since the camcorder already contains a lens but we will adopt the more common, if technically incorrect, terminology.

Lenses of 0.5 to 0.6 magnification provide a wide field of view without excessive fish-eye-style distortion. We have found low- and mid-range quality lenses more than adequate for these purposes. There are two main considerations: zoom-through capability and the amount of distortion at the edges of the image.
Some lenses focus well only at the camera's widest built-in zoom. For zooming, the wide-angle lens must be removed. In some fixed installations, zoom-through may not be necessary.

In general, the better the optics of the lens, the sharper the image will be in the corners. After compression, even nonzoom-through lenses under $\$ 30$ had acceptable image quality. Note that it is possible, with some combinations of cameras and wide-angle lenses, for the edge of the wide-angle lens to be visible in the video frame. This is more likely if the diameters of the camera and the add-on lens are not identical, requiring an adapter which may space the wide-angle lens away from the camera lens.

\section{E. Other components}

We highly recommend a live means of monitoring the recording process, if only for setup and test purposes. Regardless of specific components, it is best to monitor the output of the DVD recorder to capture the entire chain of components. Conceptually simplest is one television set and one pair of headphones connected to the outputs of each DVD recorder; options more feasible for multiple-camera setups are described in Appendix C.

A visual indication of audio recording levels is also useful. A series of light-emitting diodes (LEDs) for each audio channel gives immediate feedback that the system is recording some sound at the right level. Quality of that sound, of course, must still be verified audibly, but a link in the chain fails completely more often than a slight degradation occurs, and a visual LED system quickly highlights this. Commercial visual monitoring systems have more features, accuracy, and cost than this use requires, but a simple uncalibrated version is a common beginner's do-it-yourself electronics kit.

Since loss of power during recording may cause all data on the disc to be lost, seriously consider an uninterruptible power supply for the recorders. Actual power requirements are low, so even the most basic should be adequate.

\section{F. Physical room considerations}

The physical setup of the recording room is frequently overlooked when seeking to improve audio quality but it is in fact the most critical. While often constrained by a variety of factors, physical attributes are some of the easiest and most cost-effective variables.

An acoustically absorptive room, while the precise opposite of most typical classrooms, will improve speech intelligibility, particularly if students work in multiple small groups. As well as providing cover for cables, a dropped acoustic ceiling dramatically decreases echoes and extraneous room noises, and any amount of carpet, curtains, or even bulletin boards will further improve the situation. These will be most effective when placed behind the students, as seen by the microphone.

The most noticeable improvement to the room, however, is careful selection of the seating. Perhaps the most disruptive noise to a successful transcription is the incessant scraping and resonating of chair legs against the floor. Although 
barely noticeable live, microphones seemingly amplify this particular sound by an order of magnitude. Wheeled chairs, carpet, or other silent chair leg surfaces are well worth the investment.

Forced air heating and air conditioning systems, even from neighboring rooms, can also create enough noise to render some recordings unusable. A localized thermostat control is useful. Likewise, open windows will introduce the transcriptionist to more wind, traffic, and chirping birds than ever before noticed.

Fluorescent lights can cause electrical interference with unbalanced microphone cables, although few other lighting options are often feasible. Attempt to keep unbalanced microphone cables away from the lights and wiring, and cross at $90^{\circ}$ angles where possible. Likewise, it is best to choose a room far from heavy machinery, microwave ovens, and other possible sources of electrical interference. If forced into a room with potential interference sources, seriously consider professional microphone systems with balanced cables.

If students themselves are working with technology and a computer or television screen is to be recorded, an LCD monitor or projector can be successfully captured on camera. Scan lines will appear from a CRT or plasma display if the refresh rates are not exactly the same. Best, of course, is to use an analog output from the computer or TV directly into a DVD recorder (to record onto a separate disc) or video splitter prior to a DVD recorder (to be combined picture-inpicture with the camera's view on a single disc).

\section{GENERAL CONCLUSIONS}

Although we have discussed the use of DVD video recorders in depth, we have encountered numerous items applicable to a broad range of setups and approaches. Microphone types and arrangement, in particular, would benefit from more experimentation, and we welcome any input from the community at large.

Each research group has a distinct set of needs and constraints. Some specific recommendations are given in Appendix E, but perhaps more valuable are realistic expectations and the right approach to planning, choosing, setting up, and troubleshooting the system. To that end, these are the most important items to consider.

(1) Audio is more important and video less important than might be expected.

(2) Let the teachers teach, not fuss with cables. Once the recording quality is considered good enough to be analyzed, ease of use should prevail over all other factors. What is simple in test (e.g., replacing a battery) becomes complicated in the 5 min before a class.

(3) When developing the system, work backward from the desired output. Begin by first choosing an interface compatible with an acceptable transcription system and archiving medium, then select compatible hardware components.

(4) Thoroughly test file compatibility with the chosen transcription method or software.

(5) Test as much physical hardware as possible under identical conditions before purchasing.

(6) Do not invest in technology merely because it is newer or more advanced (e.g., digital video between camera and recorder adds significant cost and complexity for only moderate gain.)

(7) Beware of external factors such as outside noise, electrical interference, etc.

(8) A simple backup solution, such as a tape in the camcorder, can preserve at least some data in the event of a last-minute setup issue.

(9) Electrical interference in unbalanced microphone cables can prove exceedingly difficult and time consuming, if not impossible, to solve.

(10) Investments in the mundane, such as room layout, chairs, etc., can make far greater impacts than technology improvements. They are, however, more difficult to write into a grant.

\section{ACKNOWLEDGMENT}

This work has been financially supported by the Swedish Research Council, research Grant No. DNR 721-2002-3219, "To Inform a Scholarly Base for Teaching Physics at University."

\section{APPENDIX: SUPPLEMENTARY AUXILIARY MATERIAL}

See separate auxiliary material for information regarding the following: using the transcription software TRANSANA directly from DVDs; several other methods of transferring audio and video data into a computer, their advantages, disadvantages, and potential uses; the development of systems with multiple cameras; the development of systems that can be transported between rooms; and specific equipment recommendations and estimated costs for the various types of systems, for budgeting and planning purposes.

\footnotetext{
*Electronic mail: rebecca.kung@fysik.uu.se

${ }^{1}$ F. Byers, "Care and Handling of CDs and DVDs-A Guide for Librarians and Archivists," Council on Library and Information Resources and National Institute of Standards and Technology, http://www.clir.org/pubs/reports/pub121/contents.html, 2003.
}

${ }^{2}$ J. Rose, "Balanced Wiring," http://www.dplay.com/dv/balance/ balance.html, 2001. 